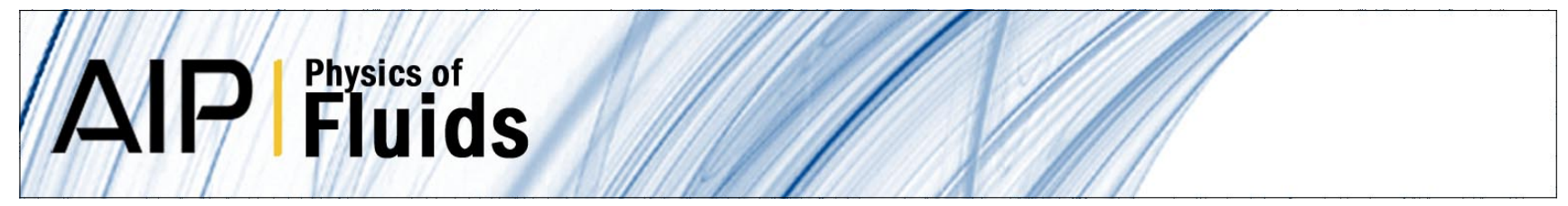

\title{
Measurement of turbulent flow upstream and downstream of a circular pipe bend
}

Jun Sakakibara and Nobuteru Machida

Citation: Phys. Fluids 24, 041702 (2012); doi: 10.1063/1.4704196

View online: http://dx.doi.org/10.1063/1.4704196

View Table of Contents: http://pof.aip.org/resource/1/PHFLE6/v24/i4

Published by the American Institute of Physics.

\section{Related Articles}

Particulate mixing in a turbulent serpentine duct

Phys. Fluids 24, 013301 (2012)

Numerical investigations of turbulent flow characteristics in helically finned pipe

Phys. Fluids 23, 125106 (2011)

ODTLES simulations of wall-bounded flows

Phys. Fluids 23, 125102 (2011)

A streamwise-constant model of turbulent pipe flow

Phys. Fluids 23, 095111 (2011)

On the spectra of nozzle-exit velocity disturbances in initially nominally turbulent, transitional jets

Phys. Fluids 23, 091702 (2011)

\section{Additional information on Phys. Fluids}

Journal Homepage: http://pof.aip.org/

Journal Information: http://pof.aip.org/about/about_the_journal

Top downloads: http://pof.aip.org/features/most_downloaded

Information for Authors: http://pof.aip.org/authors

\section{ADVERTISEMENT}

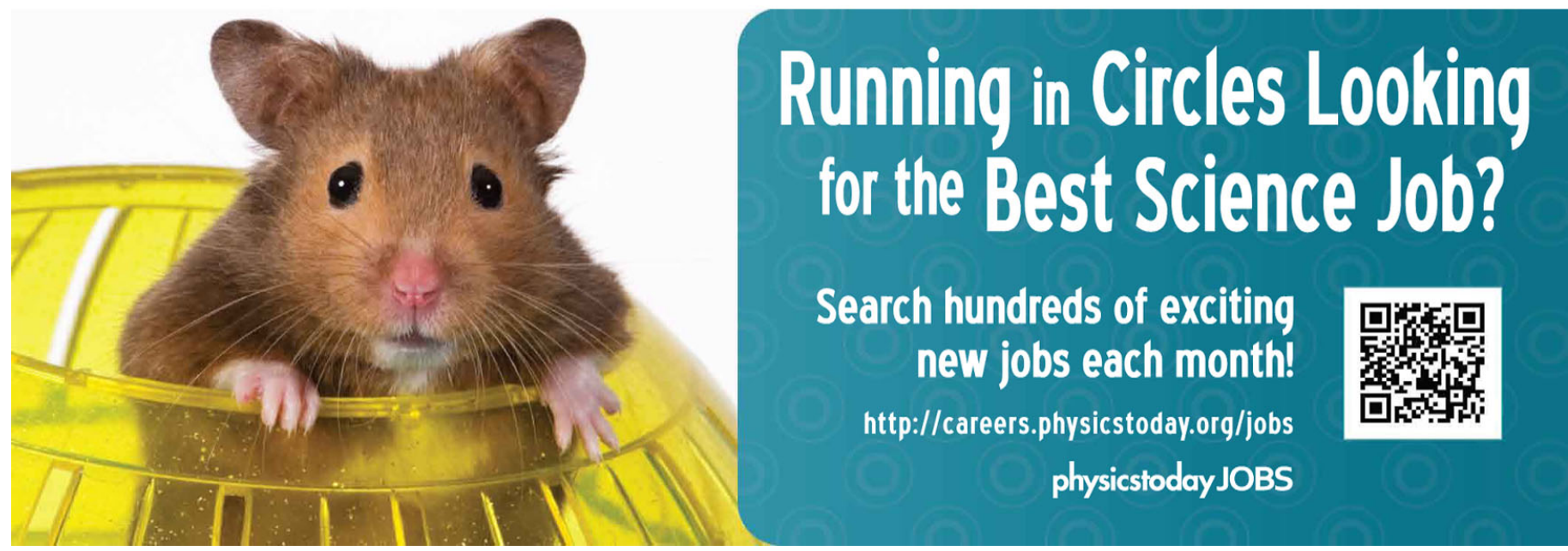




\title{
Measurement of turbulent flow upstream and downstream of a circular pipe bend
}

\author{
Jun Sakakibara and Nobuteru Machida \\ Department of Engineering Mechanics and Energy, University of Tsukuba, \\ Tsukuba 305-8573, Japan
}

(Received 10 January 2012; accepted 27 March 2012; published online 16 April 2012)

\begin{abstract}
We measured velocity distribution in cross sections of a fully developed turbulent pipe flow upstream and downstream of a $90^{\circ}$ bend by synchronizing two sets of a particle image velocimetry (PIV) system. Unsteady undulation of Dean vortices formed downstream from the bend was characterized by the azimuthal position of the stagnation point found on the inner and outer sides of the bend. Linear stochastic estimation was applied to capture the upstream flow field conditioned by the azimuthal location of the stagnation point downstream from the bend. When the inner-side stagnation point stayed below (above) the symmetry plane, the conditional streamwise velocity upstream from the bend exhibited high-speed streaks extended in a quasistreamwise direction on the outer side of the curvature above (below) the symmetry plane. (C) 2012 American Institute of Physics. [http://dx.doi.org/10.1063/1.4704196]
\end{abstract}

The flow in a pipe with a $90^{\circ}$ bend has been paid particular attention because of the complex feature of the spatially evolving flow structure downstream from the bend, ${ }^{1,2}$ in addition to the importance in engineering applications. While the flow at small Dean numbers with a laminar inlet condition consists of a pair of steady counter-rotating vortices, the flow at larger Dean numbers with turbulence exhibits unsteady and anti-symmetric behavior of the secondary flow.

Tunstall and Harvey, ${ }^{3}$ using their apparatus with a sharp corner edge at the curved section, found a secondary circulation that does not conform to the twin-circulatory flow observed in the case of a laminar small Dean number flow. The secondary flow is dominated by a single clockwise or anticlockwise circulation about the axis, between which it switches abruptly at a low frequency, which is known as 'swirl switching'.

Rutten et al. $^{4}$ computed the same flow by large eddy simulation and determined the power spectra of the overall forces onto the pipe walls. At the largest Reynolds number, they showed spectra exhibiting an oscillation at a frequency much lower than that commonly observed at vortex shedding from separation at the inner side of a bend. They also found that the low-frequency oscillation perceptible on the entire wall is caused by the two Dean vortices whose strength varies in time and which as a result alternately dominate the flow field. The domination of one of the Dean vortices normally leads to a displacement of the stagnation point into an asymmetric position. Based on the time history of the azimuthal position of the stagnation point, Rutten et al. ${ }^{4}$ pointed out that the stagnation point does not merely switch between only two stable positions, as reported by Tunstall and Harvey, ${ }^{3}$ but stays also at any angular position within $\pm 40^{\circ}$.

Tunstall and Harvey ${ }^{3}$ proposed that the swirl switching is the result of turbulent circulation that occasionally enters the bend. However, no evidence of such a proposition can be found in the literature, and the source of the unsteady movement of the stagnation point remains unclear. In our study, we tried to find a structure existing in the upstream tangent of the bend; this structure is responsible for the movement of the stagnation point in the downstream tangent. The stagnation point is identified from the velocity vector field measured at a cross section of the pipe downstream from the bend by using single-camera particle image velocimetry (PIV) and computing the conditional velocity field upstream from the bend, which is measured by additional stereo-PIV. 
The experiment was conducted with a closed-loop circuit of round pipe with a diameter $D$ $=50 \mathrm{~mm}$. The working fluid was tap water purified by cartridge filters with $25-\mu \mathrm{m}$ and $50-\mu \mathrm{m}$ pores. The centrifugal pump (MDH-401SE5-D, Iwaki) was followed by a perforated plate and a flow straightener consisting of an aluminum honeycomb, and the straight section of $80 \mathrm{D}$ in length before a $90^{\circ}$ pipe bend. The curvature radius of the center line of the bend was $R_{\mathrm{c}}=D$. The bend was followed by a straight test section $160 \mathrm{D}$ long. The pipe bend was milled from a block of Plexiglas by a computer numerical control machine. Both the upstream and downstream tangents were placed horizontally and were made of 2.5 -mm-thick Plexiglas pipe. The rest of the pipe system was constructed from polyvinyl chloride (PVC) water pipe except the upstream and downstream sides of the pump, where Tetron blade hoses, each $4 \mathrm{~m}$ long, were used to connect the pump and PVC pipe to reduce the propagation of the vibration from the pump to the circuit. The test sections were surrounded by a Plexiglas rectangular container filled with water, i.e., a water jacket, to minimize the distortion of the image observed across the round surface of the pipe. The pump was controlled by a variable-frequency drive (L100-022LFR, Hitachi), and the flow rate was monitored by an electromagnetic flow meter (AXF050G, Yokogawa). The water temperature was maintained at $20.0 \pm 0.1^{\circ} \mathrm{C}$ by use of a temperature controller (TC-3000, As-one) in conjunction with a chiller (RKS750F, Orion). The bulk Reynolds number was set at $R e=W_{b} D / v=2.7 \times 10^{4}$, where $W_{b}$ and $v$ represent the bulk velocity and kinematic viscosity, respectively. The mean and second-order properties of the flow field measured at $8 D$ upstream of the inlet of the bend are consistent with the results of numerous other pipe flow experiments. In particular, the deviation of the mean velocity profile of the present flow from that of direct numerical simulation by $\mathrm{Wu}$ and $\mathrm{Moin}^{5}$ was less than $1 \%$ in the radial range between $(R-r)^{+}=40$ and 600 , which covers the whole region of the logarithmic layer. The streamwise and radial components of root mean square velocity deviated from the data provided by the same authors by less than $10 \%$ maximum in the region of $1-r / R$ $>0.06$. Thus, we consider the present flow to be representative of typical turbulent pipe flows. We employed two rectangular Cartesian coordinates, $(x, y, z)$ and $\left(x^{\prime}, y^{\prime}, z^{\prime}\right)$, where $y$ and $y^{\prime}$ are normal to a symmetry plane of the bend, $z$ and $z^{\prime}$ are parallel to the centerline of the straight pipe downstream and upstream from the bend, respectively, $x$ is normal to both $y$ and $z$, and $x^{\prime}$ is normal to both $y^{\prime}$ and $z^{\prime}$, as shown in Fig. 1. The origin of the coordinate system of $(x, y, z)$ was set at the center of the pipe in a cross section at the end of the bend, and similarly $\left(x^{\prime}, y^{\prime}, z^{\prime}\right)$ was set at the beginning of the bend.

We used a stereoscopic PIV that is capable of resolving time-dependent, three-component velocity in a cross-sectional plane of the pipe, in conjunction with another single-camera PIV resolving two components of velocity in a different cross section, as shown in Fig. 1. The stereoPIV system consists of the following equipment: a 2-mm-thick laser light sheet was produced

(a)

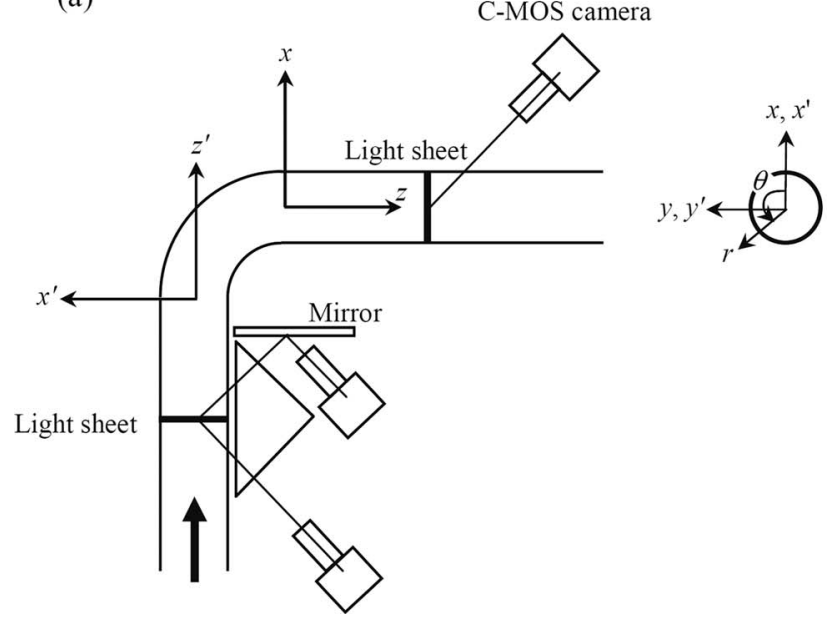

(b)

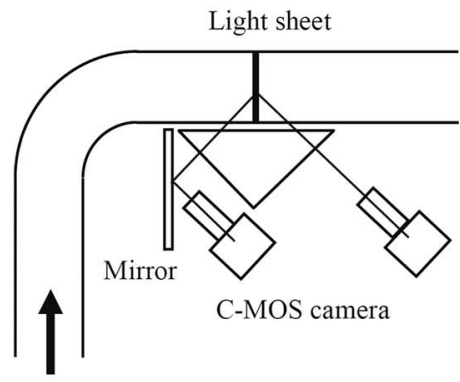

FIG. 1. Arrangement of PIV equipment. (a) Stereo-PIV upstream and single-camera PIV downstream. The coordinate system is specified. (b) Stereo-PIV downstream. 
by a neodymium-doped yttrium lithium fluoride laser (DM-10-527, $10 \mathrm{~mJ} / \mathrm{pulse}$ at $1 \mathrm{kHz}$, Photonics Industries) through a cylindrical lens to illuminate a plane normal to the flow direction. Twin high-speed complementary metal-oxide-semiconductor (C-MOS) cameras (Fastcam 1024-PCI, $1024 \times 1024$ pixels, $1000 \mathrm{fps}$ in maximum, Photron) with zoom lenses (AF Nikkor 28-105 mm, Nikon) were positioned to view the tracer particles in the same region of interest covering the whole cross section of the pipe. The aperture of the lens was set at $f / 16$. To measure the velocity field in the cross section of the pipe as close as possible to the bend, the camera placed on the upstream side captured images reflected by a vertical flat mirror, as shown in Fig. 1, while the other camera viewed the scene without a mirror. By this configuration the minimum streamwise distance from the entry, or exit, of the bend to the measurement plane was 3.1 D. The angle between the axes of observation by the two cameras was set to about $90^{\circ}$, and their lenses were mounted to satisfy the Scheimpflug condition. A triangle prism consisting of a Plexiglass water container was placed close to the water jacket to minimize astigmatic aberration of the image due to the inclined incidence of the optical axis to the test section. The single-camera PIV system consisted of another laser and camera set: a 2-mm-thick laser light sheet was produced by a Nd-YAG laser (Nano S PIV, $532 \mathrm{~nm}, 50 \mathrm{~mJ} / \mathrm{pulse}$, $20 \mathrm{~Hz}$, Litron Lasers) through a cylindrical lens to illuminate a plane normal to the flow direction. A high-speed C-MOS camera (Fastcam SA3, $1024 \times 1024$ pixels, 2000 fps maximum, Photron) with a telephoto lens (Micro Nikkor $105 \mathrm{~mm}$, Nikon) was positioned to view the tracer particles in the laser light sheet volume. The aperture of the lens was set at $f / 32$. The angle of the optical axis of the camera with respect to the light sheet was $\sim 45^{\circ}$. Since the laser was more powerful than that used in the aforementioned stereo-PIV, the aperture of the lens was much smaller (f/32), and hence the particles were imaged in good focus even though neither the Scheimpflug arrangement of the lens nor the prism behind the test section was used.

While the stereo-PIV is capable of measuring three-component velocity vectors, the singlecamera PIV can measure only two components that are normal to the optical axis of the lens, which is not always normal to the light sheet plane like the one used in this study. Since the $y$ axis is normal to the optical axis, the $v$-component can be measured directly, while the other components, such as the $u$ - and $w$-components, cannot be decomposed from the vector, because the $x$ and $z$ axes are $\sim 45^{\circ}$ with respect to the optical axis. Thus we used only the information on the $v$-component to extract the stagnation point, which is described in the following sections.

The water was seeded homogeneously with polyamide-12 tracer particles (Daiamid, $1.02 \sim 1.03 \mathrm{sp}$ gr, 40- $\mu \mathrm{m}$ average diameter, Daicel-Degussa).

The data rates of the stereo and single-camera PIV were set at $120 \mathrm{~Hz}$ and $20 \mathrm{~Hz}$, respectively. The latter is six times smaller than the former, since the maximum frequency of the laser for singlecamera PIV was limited. The camera for the stereo-PIV could typically capture 1600 successive time-dependent pairs of images for each camera at a single run of recording. To obtain well-converged statistics, we made 29 recording runs. Thus a total of 46400 realizations of the instantaneous velocity field were obtained. Both the stereo and the single-camera PIV system were synchronized by a pulse generator (Model 9600, Quantum Composers), and the particle images were recorded for the same duration of time.

PIV interrogation was performed at equally spaced Cartesian grid points at 1-mm intervals in two directions in the cross section of the pipe. The time interval separating the two PIV single exposures was set at $750 \mu \mathrm{s}$, and the mean displacement of the particle was $\sim 5$ pixels. Since the error in measuring the displacement of the tracers was within 0.1 pixel, the error of the instantaneous velocity was estimated to be $2 \%$ of the mean velocity. The spatial resolution of the velocity measurement was limited by the size of the interrogation spot. Consider a sinusoidal velocity with spatial wavelength $\Lambda$ and size $N$ of the interrogation window of the PIV. Gain $G$ of the measured velocity compared with the true velocity was derived by $\mathrm{Hart}^{6}$ as follows:

$$
G=|\Lambda \sin (\pi N / \Lambda) /(\pi N)|
$$

In the present measurements, the dimensions of the interrogation window were approximately $N_{\mathrm{x}}=2.4 \mathrm{~mm}$ (30 pixels) in the $x^{\prime}$ direction and $N_{\mathrm{y}}=1.7 \mathrm{~mm}$ (30 pixels) in the $y^{\prime}$ direction. Thus, the wavelengths at which the gain dropped by $50 \%(G=0.5)$ were computed as $4.0 \mathrm{~mm}$ and $2.8 \mathrm{~mm}$ in the $x^{\prime}$ and $y^{\prime}$ directions, respectively. This wavelength was $\sim 6-8 \%$ of the pipe diameter, and it was 


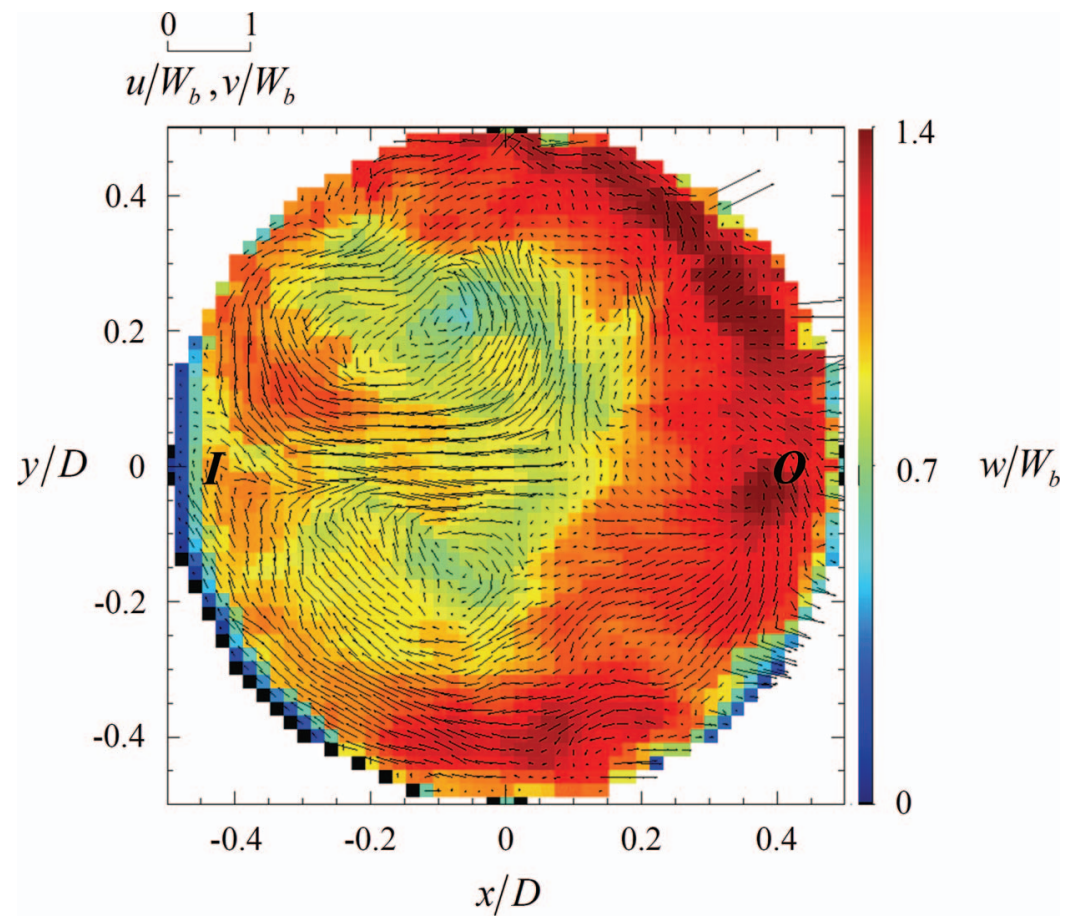

FIG. 2. Instantaneous velocity vectors in a cross section at $z=3.1 D$ downstream from the bend. Inner and outer sides are indicated by labels $I$ and $O$, respectively.

two orders of magnitude larger than the estimated Kolmogorov length scale $(\eta \sim 0.08 \mathrm{~mm})$ of the present flow. Obviously, the results shown in this paper will focus on large-scale structures without resolving small dissipative eddies.

Figure 2 shows an instantaneous velocity vector at $z=3.1 \mathrm{D}$ downstream from the bend. The color contour represents the streamwise component of the velocity. It is clearly shown that counter-rotating Dean vortices are created. The center of the vortices are located above and below the symmetry plane, i.e., the plane at $y=0$, and a strong inflow is induced between vortices towards positive $x$. The flow near the wall on the upper (lower) side circulates mostly counterclockwise (clockwise) and forms a stagnation point near $(x, y)=(-0.45 \mathrm{D}, 0.1 \mathrm{D})$ at which the fluid starts to eject towards the central part of the pipe. Rutten et al. ${ }^{4}$ identified the stagnation point as the minimum azimuthal and radial components of spatially low-pass-filtered instantaneous velocity vectors at the circle slightly above the wall of the pipe. In our study, however, the radial component of the velocity vectors could not be measured independently from other components, as mentioned previously. Thus, we simply used the $v$-component of velocity only to determine the stagnation point.

Figure 3 shows the temporal variation of the $v$-component of velocity vectors on a circle of $r=0.88 R$ at $z / D=3.1$, where $R$ represents the pipe radius $(=D / 2)$. The vertical axis is extended above $3 \pi$ for clarity. It is understood that the relatively clear gap between positive and negative values of $v$, i.e., the boundary between red and blue in the figure, near $\theta=\pi$, represents the innerside (negative $x$ ) stagnation point. Another gap, found near $\theta=2 \pi$, might represent the outer-side stagnation point. Since the small-scale turbulence is superposed on the large-scale Dean vortices, some low-pass filtering is necessary to identify the stagnation point at which $v$ equals zero, as stated by Rutten $\mathrm{et} \mathrm{al}^{4}{ }^{4}$ We established the following method to identify the stagnation point: The azimuthal variation of $v$ is sinusoidal, and its phase is expected to vary along with the movement of the stagnation point. To find the phase, we calculated the correlation function between $\sin$ and $v$ as follows:

$$
c(\eta)=\left[\frac{\int_{0}^{2 \pi}(v-\bar{v}) \cdot \sin (2 \theta-\eta) d \theta}{\int_{0}^{2 \pi}(v-\bar{v})^{2} d \theta}\right]^{1 / 2} \text { where } \bar{v}=\frac{1}{2 \pi} \int_{0}^{2 \pi} v d \theta
$$




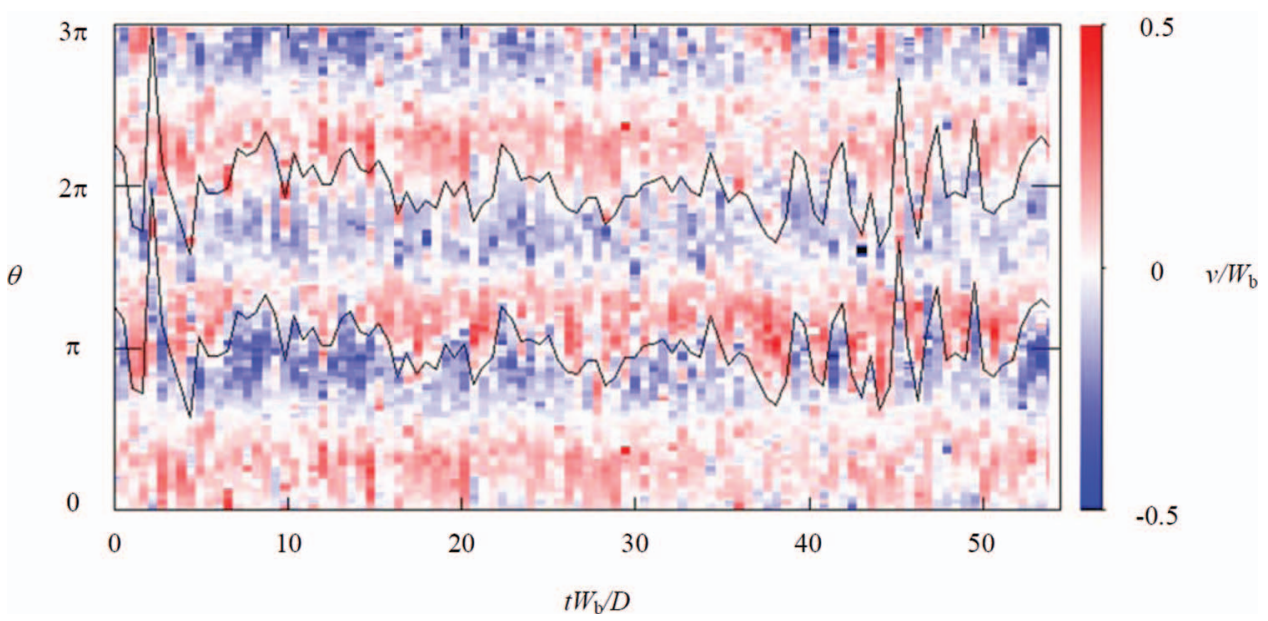

FIG. 3. Temporal variation of the $v$-component of instantaneous velocity vectors on a circle of $r=0.88 R$ measured by the single-camera PIV. The solid line represents the stagnation point, i.e., the location where $v=0$, estimated by fitting sin function.

and we then found the phase $\eta=\eta_{m}$ at which the correlation coefficient $c$ has the maximum. Thus, the inner and outer sides of the stagnation point are determined as follows:

$$
\theta_{\text {in }}=\pi-\eta_{m} \text { and } \theta_{\text {out }}=-\eta_{m},
$$

respectively. The solid line in Fig. 3 shows both the inner- and outer-side stagnation points. The inner stagnation point reasonably represents the boundary between the positive and negative regions of $v$, while the outer stagnation point is more random. Note that the standard deviation of $\theta_{\text {in }}$ was $\sigma\left(\theta_{\text {in }}\right)=33.1^{\circ}$.

The linear stochastic estimation ${ }^{7}$ of the conditional velocity field upstream from the bend was computed based on the azimuthal location of the inner stagnation point as the conditional event. The conditional average of the $i$-th component of the velocity vector at position $\mathbf{x}=\left(x^{\prime}, y^{\prime}, z^{\prime}\right)$ and time $t^{\prime}$ based on the azimuthal location of the stagnation point at time $t$, as a conditional event, is written as $\left\langle u_{i}\left(\mathbf{x}, t^{\prime}\right) \mid \theta_{i n}(t)\right\rangle$. The equation for linear stochastic estimations of $\left\langle u_{i}\left(\mathbf{x}, t^{\prime}\right) \mid \theta_{i n}(t)\right\rangle$ is

$$
\hat{u}_{i}\left(\mathbf{x}, t^{\prime}, t\right)=\text { linear estimate of }\left\langle u_{i}\left(\mathbf{x}, t^{\prime}\right) \mid \theta_{i n}(t)\right\rangle=L_{i}\left(\mathbf{x}, t^{\prime}-t\right) \theta_{i n}(t) .
$$

The manipulation to minimize the mean square error of the estimate leads to a linear algebraic equation for the estimation coefficients $L_{i}$,

$$
L_{i}=\left\langle\theta_{\text {in }}(t) u_{i}(\mathbf{x}, t+\tau)\right\rangle /\left\langle\theta_{i n}^{2}(t)\right\rangle,
$$

where $\tau=t^{\prime}-t$ when the flow field is statistically homogeneous in time, and \langle\rangle denotes the ensemble average. Thus the conditional velocity under the event $\theta_{\text {ref }}$ is given by

$$
\hat{u}_{i}(\mathbf{x}, \tau)=L_{i} \theta_{\text {ref }} .
$$

Figure 4 shows the streamwise component of conditional fluctuating velocity vectors, $\hat{w}$, at $z^{\prime}=-3.1 D$ upstream from the bend. The event was set at $\theta_{\text {ref }}=\left\langle\theta_{i n}^{2}\right\rangle^{1 / 2}$, i.e., the stagnation point was located one standard deviation of its variation below (toward the negative $y$ direction) the symmetry plane. Based on Taylor's frozen turbulence hypothesis, time lag $\tau$ was converted into the streamwise distance $z^{\prime *}=-\tau W_{b}$, where the convection velocity of eddies was assumed to be identical to the bulk velocity $W_{b}$. It is clear that the high (low) speed streaks extending in a quasi-streamwise direction are found at the outer side, i.e., $x^{\prime}>0$, above (below) the symmetry plane. The most significant streaks appeared first at $z^{\prime *}=7 \mathrm{D}$, which is comparable to the distance $l$ measured from the upstream to the downstream location of the measurement along the centerline of the pipe, $l=7.77 D$. The streaks have a slight inclination with respect to the $x^{\prime}-z^{\prime *}$ plane and get closer to one another downstream. The streamwise extension of the streaks is $\sim 5 D$. 


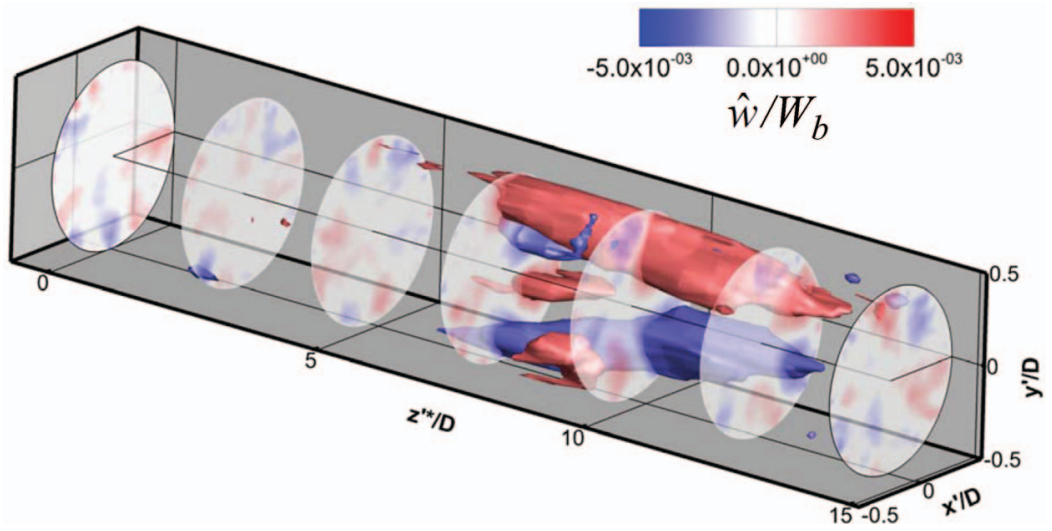

FIG. 4. Streamwise component of conditional fluctuating velocity vectors, $\hat{w}$, at $z^{\prime}=-3.1 D$ upstream from the bend. The value is normalized by $W_{b}$. The surfaces of the constant value of $\hat{w}= \pm 0.003 W_{b}$ are superposed.

It is also noticeable that another pair of the streaks, but with the opposite sign, is extended over $z^{\prime *}=7 D-8 D$. Since the streaks appeared prior to the Dean motion, i.e., $t^{\prime}<t$, the upstream propagation of the pressure wave induced by the Dean motion has no chance of contributing to the formation of the streak. Thus, it is reasonable to expect that the streaks, or their effects, travel downstream with a convection velocity similar to the bulk velocity and reach the downstream measurement location, where the azimuthal displacement of the stagnation point is detected.

Figure 5 shows the conditional vectors in a planar section at $z^{*}=10 \mathrm{D}$, where the velocity magnitude of the streak is most intense. An in-plane vector plot reveals that the radial flow towards the wall is observed in the high-speed streak (red part), and the inward flow is observed in the low-speed streak, forming a streamwise eddy centered in between the streaks. This is consistent with a momentum exchange between the high- and low-speed regions in the boundary layer due to the flow induced by streamwise eddies. The magnitude of the conditional streamwise velocity of the streaks reaches $\sim 0.01 W_{b}$, which corresponds to $0.2 u_{\tau}$, where $u_{\tau}$ denotes friction velocity. Note

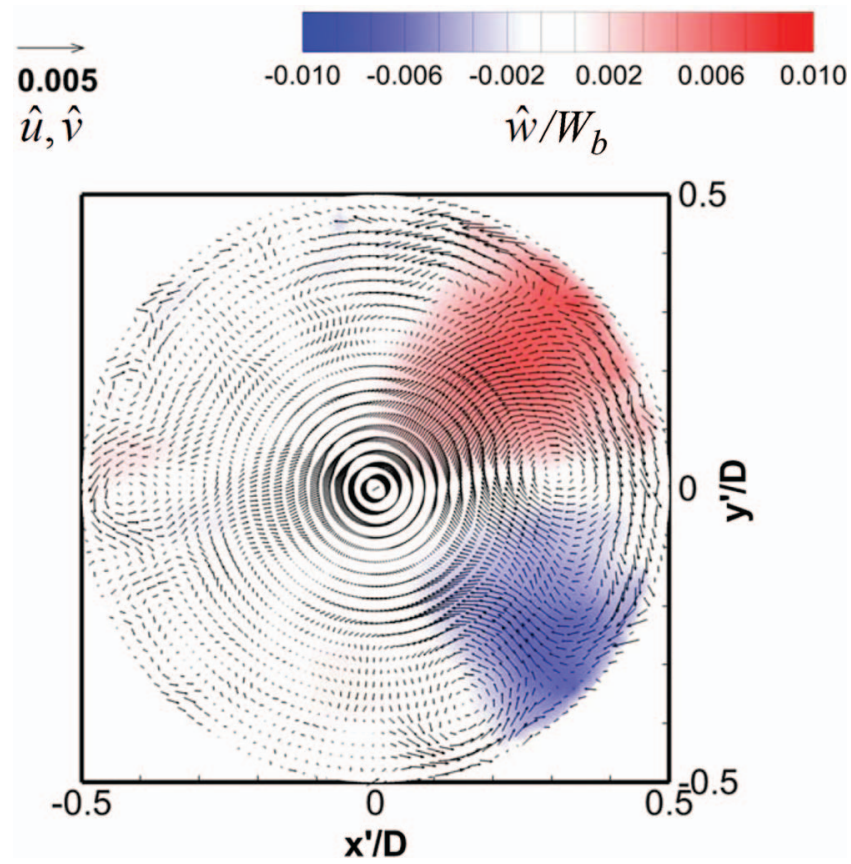

FIG. 5. Conditional fluctuating velocity vectors at $z^{\prime *}=10 \mathrm{D}$. The value is normalized by $W_{b}$. 
that the cross-correlation coefficient of $\theta_{\text {in }}$ and $w^{\prime}$ was $\sim 0.1$ at maximum. This value is comparable to or even larger than the two-point correlation coefficient of the streamwise velocity component in a turbulent pipe flow separated by the distance, matching the present measurement locations. ${ }^{5}$

Kim and Adrian ${ }^{8}$ suggested the existence of very large scale motion (VLSM) in the form of a large streamwise extent in the logarithmic layer. The streamwise length of the structure was found to be $14 R,{ }^{8}$ or even longer, like $25 R,{ }^{9}$ and the meandering motion of such structures is evident. ${ }^{9,10}$ The present streaks are shorter in the streamwise length than the VLSM, and the value of the conditional streamwise velocity $\left(\sim 0.2 u_{\tau}\right)$ is one order lower than the typical magnitude of the VLSM, which is $\sim 3 u_{\tau}$, as shown in Ref. 9 .

One of reasons for this discrepancy is that the present streaks were estimated in the sense of conditional averaging of large numbers of realizations, which might smooth out the meandering feature of the instantaneous structures of VLSM; consequently, the streamwise length and velocity amplitude could be reduced. While the connections between VLSM and the streaks are unclear at this stage, there is little doubt the estimated streaks are responsible for bringing the inner-side stagnation point to the negative $y$ direction.

This work was partly supported by Tokyo Electric Power Company. The authors are thankful to Mr. S. Onohara and Mr. Y. Namiki for their contributions in carrying out the experiment.

${ }^{1}$ S. A. Berger and L. Talbot, "Flow in curved pipes," Ann. Rev. Fluid Mech. 15, 461-512 (1983).

${ }^{2}$ H. Ito, "Flow in curved pipes," JSME Int. J. 30, 543-552 (1987).

${ }^{3}$ M. J. Tunstall and J. K. Harvey, "On the effect of a sharp bend in a fully developed turbulent pipe-flow," J. Fluid Mech. 34, 595-608 (1968).

${ }^{4}$ F. Rutten, W. Schroder, and M. Meinke, "Large-eddy simulation of low frequency oscillations of the Dean vortices in turbulent pipe bend flows," Phys. Fluids 17, 035107 (2005).

${ }^{5} \mathrm{X}$. Wu and P. Moin, "A direct numerical simulation study on the mean velocity characteristics in turbulent pipe flow," J. Fluid Mech. 608, 81-112 (2008).

${ }^{6}$ D. P. Hart, "PIV error correction," Exp. Fluids 29, 13 (2000).

${ }^{7}$ R. J. Adrian, "Stochastic estimation of conditional structure: a review," Appl. Sci. Res. 53, 291 (1994).

${ }^{8}$ K. C. Kim and R. J. Adrian, "Very large-scale motion in the outer layer," Phys. Fluids 11, 417-422 (1999).

9 J. P. Monty, J. A. Stewart, R. C. Williams, and M. S. Chong, "Large-scale features in turbulent pipe and channel flows," J. Fluid Mech. 589, 147-156 (2007).

${ }^{10}$ L. H. O. Hellström, A. Sinha, and A. J. Smits, "Visualizing the very-large-scale motions in turbulent pipe flow," Phys. Fluids 23, 011703 (2011). 